\title{
Multi-point boundary value problems for lower semicontinuous differential inclusions
}

\author{
Mouffak Benchohra and Sotiris K. Ntouyas
}




\title{
MULTI-POINT BOUNDARY VALUE PROBLEMS FOR LOWER SEMICONTINUOUS DIFFERENTIAL INCLUSIONS
}

\author{
Mouffak Benchohra \\ Laboratoire de Mathématiques, Université de Sidi Bel Abbès \\ BP 89, 22000 Sidi Bel Abbès, Algérie \\ benchohra@yahoo.com \\ SotiRis K. NTOUYAS \\ Department of Mathematics, University of Ioannina \\ 45110 Ioannina, Greece \\ sntouyas@cc.uoi.gr
}

[Received: March 3, 2002]

\begin{abstract}
In this paper, Schaefer's fixed point theorem combined with a selection theorem due to Bressan and Colombo is used to investigate the existence of solutions to multi-point boundary value problems for second order differential inclusions with lower semicontinuous and nonconvex valued right-hand side.
\end{abstract}

Mathematical Subject Classification: 34A60, 34B10, 34B15

Keywords: multi-point boundary value problems, measurable selection, existence, fixed point, decomposable valued map.

\section{Introduction}

In this paper we shall prove existence results for multi-point boundary value problems (BVP for short). In Section 3 we study the three-point boundary value problem of the second order differential inclusion

$$
\begin{gathered}
y^{\prime \prime} \in F(t, y), \quad t \in J=[0,1] \\
y(0)=0, y(\eta)=y(1),
\end{gathered}
$$

where $F: J \times \mathbb{R} \longrightarrow \mathcal{P}(\mathbb{R})$ is a given multivalued map, $\eta \in(0,1)$ and $\mathcal{P}(\mathbb{R})$ is the family of all subsets in $\mathbb{R}$.

Section 4 is devoted to studying the following four-point BVP

$$
\begin{gathered}
y^{\prime \prime} \in F(t, y), \quad t \in J=[0,1] \\
y(0)=y^{\prime}(\eta), y(1)=y(\tau),
\end{gathered}
$$

where $F, \eta$ are as in the problem (1.1)-(1.2) and $\tau \in(0,1)$.

The boundary value problems (1.1)-(1.2) and (1.3)-(1.4) in the case when the multivalued $F$ has convex values were studied very recently by Benchohra and Ntouyas 
in [1], [3] by using a fixed point theorem for condensing multivalued maps due to Martelli [18]. The above problems in the case when the multivalued $F$ has nonconvex values were studied by the same authors in [2], with the aid of a fixed point theorem for contraction multivalued maps due to Covitz and Nadler [7]. Here, by using Schaefer's fixed point theorem, combined with a selection theorem of Bressan and Colombo for lower semicontinuous and nonconvex valued multivalued operators with decomposable values, existence results are proposed for the problems (1.1)-(1.2) and (1.3)-(1.4). Possible generalizations are indicated in Section 4. For recent results on multi-point BVP for ordinary differential equations we refer to [11]-[14].

\section{Preliminaries}

In this section, we introduce notations, definitions, and preliminary facts from multivalued analysis which are used throughout this paper.

By $C(J, \mathbb{R})$ we denote the Banach space of all continuous functions from $J$ into $\mathbb{R}$ with the norm

$$
\|y\|_{\infty}:=\sup \{|y(t)|: t \in J\} .
$$

$L^{1}(J, \mathbb{R})$ denotes the Banach space of measurable functions $y: J \longrightarrow E$ which are Lebesgue integrable normed by

$$
\|y\|_{L^{1}}=\int_{0}^{b}|y(t)| d t \quad \text { for all } \quad y \in L^{1}(J, \mathbb{R}) .
$$

$A C^{i}([0, T], \mathbb{R})$ is the space of $i$-times differentiable functions $y:[0, T] \rightarrow \mathbb{R}$, whose $i^{t h}$ derivative, $y^{(i)}$, is absolutely continuous.

Let $A$ be a subset of $J \times \mathbb{R}$. $A$ is $\mathcal{L} \otimes \mathcal{B}$ measurable if $A$ belongs to the $\sigma$-algebra generated by all sets of the form $\mathcal{I} \times D$ where $\mathcal{I}$ is Lebesgue measurable in $J$ and $D$ is Borel measurable in $\mathbb{R}$. A subset $B$ of $L^{1}(J, \mathbb{R})$ is decomposable if, for all $u, v \in B$ and $\mathcal{I} \subset J$ measurable, the function $u \chi_{\mathcal{I}}+v \chi_{J-\mathcal{I}} \in B$, where $\chi$ denotes the characteristic function.

Let $E$ be a separable Banach space, $X$ a nonempty closed subset of $E$ and $G$ : $X \rightarrow \mathcal{P}(E)$ a multivalued operator with nonempty closed values. $G$ is lower semicontinuous (l.s.c.) if the set $\{x \in X: G(x) \cap C \neq \emptyset\}$ is open for any open set $C$ in $E$. $G$ has a fixed point if there is $x \in X$ such that $x \in G(x)$.

Definition 1. Let $Y$ be a separable metric space and let $N: Y \rightarrow \mathcal{P}\left(L^{1}(J, \mathbb{R})\right)$ be a multivalued operator. We say $N$ has the property $(B C)$ if

1) $N$ is lower semi-continuous (l.s.c.);

2) $N$ has nonempty closed and decomposable values.

Let $F: J \times \mathbb{R} \rightarrow \mathcal{P}(\mathbb{R})$ be a multivalued map with nonempty compact values. Assign to $F$ the multivalued operator

$$
\mathcal{F}: C(J, \mathbb{R}) \rightarrow \mathcal{P}\left(L^{1}(J, \mathbb{R})\right)
$$


by letting

$$
\mathcal{F}(y)=\left\{w \in L^{1}(J, \mathbb{R}): w(t) \in F(t, y(t)) \text { for a.e. } t \in J\right\} .
$$

The operator $\mathcal{F}$ is called the Niemytzki operator associated with $F$. We say $F$ is the lower semi-continuous type (l.s.c. type) if its associated Niemytzki operator $\mathcal{F}$ is lower semi-continuous and has nonempty closed and decomposable values.

Next we state a selection theorem due to Bressan and Colombo.

Lemma 1. [6] Let $Y$ be separable metric space and let $N: Y \rightarrow \mathcal{P}\left(L^{1}(J, \mathbb{R})\right)$ be a multivalued operator which has the property $(B C)$. Then $N$ has a continuous selection, i.e. there exists a continuous function (single-valued) $g: Y \rightarrow L^{1}(J, \mathbb{R})$ such that $g(y) \in N(y)$ for every $y \in Y$.

For more details on multivalued maps and the proof of known results cited in this section we refer to the books by Deimling [8], Gorniewicz [10], Hu and Papageorgiou [15] and Tolstonogov [20].

\section{Main Results}

By the help of Schaefer's theorem, combined with the selection theorem of Bressan and Colombo for lower semicontinuous maps with decomposable values, we shall present first an existence result for the problem (1.1)-(1.2). Before this, let us introduce the following hypotheses which are assumed hereafter:

(H1) $F:[0,1] \times \mathbb{R} \longrightarrow \mathcal{P}(\mathbb{R})$ is a nonempty compact valued multivalued map such that:

a) $(t, y) \mapsto F(t, y)$ is $\mathcal{L} \otimes \mathcal{B}$ measurable;

b) $y \mapsto F(t, y)$ is lower semi-continuous for a.e. $t \in[0,1]$;

(H2) $F$ is integrably bounded, that is, there exists a function $H \in L^{1}(J, \mathbb{R})$ such that

$\|F(t, y)\|:=\sup \{\|v\|: v \in F(t, y)\} \leq H(t)$ for almost all $t \in J$ and all $y \in \mathbb{R}$.

In the proof of our following theorem we will need for the auxiliary result:

Lemma 2. [9]. Let $F: J \times \mathbb{R} \rightarrow \mathcal{P}(\mathbb{R})$ be a multivalued map with nonempty, compact values. Assume (H1) and (H2) hold. Then $F$ is of l.s.c. type.

Definition 2. A function $y \in A C^{1}(J, \mathbb{R})$ is called a solution to the $B V P$ (1.1)-(1.2) if $y$ satisfies the differential inclusion (1.1) a.e. on $J$ and the condition (1.2).

Theorem 1. Suppose that hypotheses (H1), (H2) hold. Then the boundary value problem (1.1)-(1.2) has at least one solution.

Proof. (H1) and (H2) imply by Lemma 2 that $F$ is of the lower semi-continuous type. Then from Lemma 1 there exists a continuous function $g: C(J, \mathbb{R}) \rightarrow L^{1}(J, \mathbb{R})$ such that $g(y) \in \mathcal{F}(y)$ for all $y \in C(J, \mathbb{R})$.

We consider the problem

$$
y^{\prime \prime}(t)=g(y)(t), \text { a.e. } t \in J=[0,1],
$$




$$
y(0)=0, y(\eta)=y(1) .
$$

Remark 1. If $y \in C(J, \mathbb{R})$ is a solution to the problem (3.1)-(3.2), then $y$ is a solution to the problem (1.1)-(1.2).

Transform problem (3.1)-(3.2) into a fixed point problem. Consider the operator, $N: C(J, \mathbb{R}) \longrightarrow C(J, \mathbb{R})$ defined by:

$$
\begin{aligned}
N(y)(t)= & \int_{0}^{t}(t-s) g(y)(s) d s+\frac{t}{1-\eta} \int_{0}^{\eta}(\eta-s) g(y)(s) d s \\
& -\frac{t}{1-\eta} \int_{0}^{1}(1-s) g(y)(s) d s .
\end{aligned}
$$

We shall show that $N$ is a compact operator.

Step 1: $N$ is continuous.

Let $\left\{y_{n}\right\}$ be a sequence such that $y_{n} \rightarrow y$ in $C(J, \mathbb{R})$. Then

$$
\begin{aligned}
\left|N\left(y_{n}\right)(t)-N(y)(t)\right|= & \int_{0}^{t}(t-s)\left|g\left(y_{n}\right)(s)-g(y)(s)\right| d s \\
& +\frac{t}{1-\eta} \int_{0}^{\eta}(\eta-s)\left|g\left(y_{n}\right)(s)-g(y)(s)\right| d s \\
& -\frac{t}{1-\eta} \int_{0}^{1}(1-s)\left|g\left(y_{n}\right)(s)-g(y)(s)\right| d s .
\end{aligned}
$$

Since $g$ is continuous, then

$$
\left\|N\left(y_{n}\right)-N(y)\right\|_{\infty} \rightarrow 0 \quad \text { as } \quad n \rightarrow \infty .
$$

Step 2: $N$ is bounded on bounded sets of $C(J, \mathbb{R})$.

Indeed, it is enough to show that there exists a positive constant $c$ such that for each $h \in N(y), y \in B_{r}=\left\{y \in C(J, \mathbb{R}):\|y\|_{\infty} \leq r\right\}$ one has $\|h\|_{\infty} \leq c$.

By (H2) we have for each $t \in J$ that

$$
|h(t)| \leq \int_{0}^{t} H(s) d s+\frac{1}{1-\eta} \int_{0}^{\eta}(\eta-s) H(s) d s+\frac{1}{1-\eta} \int_{0}^{1}(1-s) H(s) d s .
$$

Then

$$
\|h\|_{\infty} \leq \int_{0}^{1} H(s) d s+\frac{1}{1-\eta} \int_{0}^{\eta}(\eta-s) H(s) d s+\frac{1}{1-\eta} \int_{0}^{1}(1-s) H(s) d s:=c .
$$

Step 3: $N$ sends bounded sets of $C(J, \mathbb{R})$ into equicontinuous sets. 
Let $t_{1}, t_{2} \in J, t_{1}<t_{2}$ and $B_{r}$ be a bounded set of $C(J, \mathbb{R})$. Then we obtain

$$
\begin{aligned}
\left|h\left(t_{2}\right)-h\left(t_{1}\right)\right| & \leq \int_{0}^{t_{2}}\left(t_{2}-s\right)|g(y)(s)| d s+\int_{t_{1}}^{t_{2}}\left(t_{1}-s\right)|g(y)(s)| d s \\
& +\frac{t_{2}-t_{1}}{1-\eta} \int_{0}^{\eta}(\eta-s)|g(y)(s)| d s+\frac{t_{2}-t_{1}}{1-\eta} \int_{0}^{1}(1-s)|g(y)(s)| d s \\
& \leq \int_{0}^{t_{2}}\left(t_{2}-s\right) H(s) d s+\int_{t_{1}}^{t_{2}}\left(t_{1}-s\right) H(s) d s \\
& +\frac{t_{2}-t_{1}}{1-\eta} \int_{0}^{\eta}(\eta-s) H(s) d s+\frac{t_{2}-t_{1}}{1-\eta} \int_{0}^{1}(1-s) H(s) d s .
\end{aligned}
$$

As $t_{2} \longrightarrow t_{1}$ the right-hand side of the above inequality tends to zero.

As a consequence of Steps 1 to 3, together with the Arzela-Ascoli theorem we can conclude that $N$ is completely continuous.

In order to apply Schaefer's theorem, it remains to show that

Step 4: The set

$$
\Omega:=\{y \in C(J, \mathbb{R}): \lambda y=N(y) \text { for some } \lambda>1\}
$$

is bounded.

Let $y \in \Omega$. Then $\lambda y=N(y)$ for some $\lambda>1$ and

$$
\begin{aligned}
y(t) & =\lambda^{-1} \int_{0}^{t}(t-s) g(y)(s) d s+\lambda^{-1} \frac{t}{1-\eta} \int_{0}^{\eta}(\eta-s) g(y)(s) d s \\
& -\lambda^{-1} \frac{t}{1-\eta} \int_{0}^{1}(1-s) g(y)(s) d s, \quad t \in J .
\end{aligned}
$$

This implies by (H2) that for each $t \in J$ we have

$$
|y(t)| \leq \int_{0}^{t}(t-s) H(s) d s+\frac{1}{1-\eta} \int_{0}^{\eta}(\eta-s) H(s) d s+\frac{1}{1-\eta} \int_{0}^{1}(1-s) H(s) d s .
$$

Thus

$\|y\|_{\infty} \leq \int_{0}^{1}(1-s) H(s) d s+\frac{1}{1-\eta} \int_{0}^{\eta}(\eta-s) H(s) d s+\frac{1}{1-\eta} \int_{0}^{1}(1-s) H(s) d s:=K$.

This shows that $\Omega$ is bounded.

As a consequence of Schaefer's theorem (see [19] p. 29) we deduce that $N$ has a fixed point which is a solution to (3.1)-(3.2) and hence from Remark 1 a solution to the problem (1.1)-(1.2).

The next result concerns the four-point BVP (1.3)-(1.4). Before stating and proving this result, we give the definition of a solution of the four-point BVP (1.3)-(1.4).

Definition 3. A function $y \in A C^{1}(J, \mathbb{R})$ is called a solution to the BVP (1.3)-(1.4) if $y$ satisfies the differential inclusion (1.3) a.e. on $J$ and the conditions (1.4). 
Theorem 2. Assume that (H1), (H2) are satisfied. Then the BVP (1.3)-(1.4) has at least one solution on $J$.

Proof. (H1) and (H2) imply by Lemma 2 that $F$ is of the lower semi-continuous type. Then from Lemma 1 there exists a continuous function $g: C(J, \mathbb{R}) \rightarrow L^{1}(J, \mathbb{R})$ such that $g(y) \in \mathcal{F}(y)$ for all $y \in C(J, \mathbb{R})$.

We consider the problem

$$
\begin{gathered}
y^{\prime \prime}(t)=g(y)(t), \text { a.e. } t \in J=[0,1], \\
y(0)=y^{\prime}(\eta), y(1)=y(\tau) .
\end{gathered}
$$

Transform problem (3.3) - (3.4) into a fixed point problem. Consider the multivalued map, $N_{1}: C(J, \mathbb{R}) \longrightarrow C(J, \mathbb{R})$ defined by:

$$
\begin{aligned}
N_{1}(y)(t) & =\int_{0}^{t}(t-s) g(y)(s) d s+\int_{0}^{\eta} g(y)(s) d s \\
& +\frac{1+t}{1-\tau}\left[\int_{0}^{\tau}(\tau-s) g(y)(s) d s-\int_{0}^{1}(1-s) g(y)(s) d s\right] .
\end{aligned}
$$

We can easily show that $N_{1}$ has a fixed point, following the steps of Theorem 1 . We omit the details.

\section{Concluding Remarks}

Let $a_{i} \in \mathbb{R}$, with all of the $a_{i}^{\prime} s$ having the same sign, $\xi_{i} \in(0,1), i=1,2, \ldots, m-2$, $0<\xi_{1}<\xi_{2}<\ldots<\xi_{m-2}<1$. Consider the following m-point boundary value problem for second order differential inclusions

$$
\begin{gathered}
y^{\prime \prime}(t) \in F(t, y(t)), \quad t \in J, \\
y(0)=0, \quad y(1)=\sum_{i=1}^{m-2} a_{i} y\left(\xi_{i}\right) .
\end{gathered}
$$

It is well known (see [16] for example), that if a function $y \in C^{1}(J, \mathbb{R})$ satisfies the boundary condition (4.2) and all of the $a_{i}, i=1,2, \ldots, m-2$ have the same sign, then there exists $\eta \in\left[\xi_{1}, \xi_{m-2}\right]$, depending on $y \in C^{1}(J, \mathbb{R})$ such that

$$
y(1)=\alpha y(\eta),
$$

with $\alpha=\sum_{i=1}^{m-2} a_{i}$. Accordingly, the problem of the existence of a solution to the BVP (4.1)-(4.2) can be studied via the three-point BVP

$$
\begin{gathered}
y^{\prime \prime}(t) \in F(t, y(t)), \quad t \in J, \\
y(0)=0, \quad y(1)=\alpha y(\eta),
\end{gathered}
$$

where $\eta \in(0,1)$ is given. We omit the details, since the proof follows the steps of the proof of Theorem 1, with obvious modifications. 
It is obvious that the above method can also be applied to other types of m-point BVPs. For example for the BVPs

or

$$
\left\{\begin{array}{l}
y^{\prime \prime}(t) \in F(t, y(t)), \quad t \in J, \\
y^{\prime}(0)=0, \quad y(1)=\sum_{i=1}^{m-2} a_{i} y\left(\xi_{i}\right),
\end{array}\right.
$$

$$
\left\{\begin{array}{l}
y^{\prime \prime}(t) \in F(t, y(t)), \quad t \in J, \\
y(0)=0, \quad y^{\prime}(1)=\sum_{i=1}^{m-2} a_{i} y^{\prime}\left(\xi_{i}\right),
\end{array}\right.
$$

which can be reduced to the following three point BVPs:

$$
\left\{\begin{array}{l}
y^{\prime \prime}(t) \in F(t, y(t)), \quad t \in J \\
y^{\prime}(0)=0, \quad y(1)=\alpha y(\eta)
\end{array}\right.
$$

and

respectively.

$$
\left\{\begin{array}{l}
y^{\prime \prime}(t) \in F(t, y(t)), \quad t \in J \\
y(0)=0, y^{\prime}(1)=\alpha y^{\prime}(\eta)
\end{array}\right.
$$

Let $b_{j} \in \mathbb{R}$ having the same sign, $\zeta_{i} \in(0,1), i=1,2, \ldots, n-2,0<\zeta_{1}<\zeta_{2}<\ldots<$ $\zeta_{n-2}<1$. Then the following general m-point BVP

$$
\begin{aligned}
& \left\{\begin{array}{l}
y^{\prime \prime}(t) \in F(t, y(t)), \quad t \in J, \\
y(0)=\sum_{i=1}^{m-2} a_{i} y^{\prime}\left(\xi_{i}\right), \quad y(1)=\sum_{j=1}^{n-2} b_{j} y\left(\zeta_{j}\right),
\end{array}\right. \\
& \left\{\begin{array}{l}
y^{\prime \prime}(t) \in F(t, y(t)), \quad t \in J, \\
y(0)=\sum_{i=1}^{m-2} a_{i} y^{\prime}\left(\xi_{i}\right), \quad y^{\prime}(1)=\sum_{j=1}^{n-2} b_{j} y^{\prime}\left(\zeta_{j}\right),
\end{array}\right. \\
& \left\{\begin{array}{l}
y^{\prime \prime}(t) \in F(t, y(t)), \quad t \in J, \\
y(0)=\sum_{i=1}^{m-2} a_{i} y\left(\xi_{i}\right), \quad y(1)=\sum_{j=1}^{n-2} b_{j} y\left(\zeta_{j}\right)
\end{array}\right.
\end{aligned}
$$

and

$$
\left\{\begin{array}{l}
y^{\prime \prime}(t) \in F(t, y(t)), \quad t \in J, \\
y(0)=\sum_{i=1}^{m-2} a_{i} y\left(\xi_{i}\right), \quad y^{\prime}(1)=\sum_{j=1}^{n-2} b_{i} y^{\prime}\left(\zeta_{j}\right)
\end{array}\right.
$$

which can be reduced to the following four point BVP

and

$$
\begin{gathered}
\left\{\begin{array}{l}
y^{\prime \prime}(t) \in F(t, y(t)), \quad t \in J, \\
y(0)=\alpha y^{\prime}(\eta), y(1)=\beta y(\tau),
\end{array}\right. \\
\left\{\begin{array}{l}
y^{\prime \prime}(t) \in F(t, y(t)), \quad t \in J, \\
y(0)=\alpha y^{\prime}(\eta), y^{\prime}(1)=\beta y^{\prime}(\tau),
\end{array}\right. \\
\left\{\begin{array}{l}
y^{\prime \prime}(t) \in F(t, y(t)), \quad t \in J, \\
y(0)=\alpha y(\eta), y(1)=\beta y(\tau),
\end{array}\right.
\end{gathered}
$$

$$
\left\{\begin{array}{l}
y^{\prime \prime}(t) \in F(t, y(t)), \quad t \in J \\
y(0)=\alpha y(\eta), \quad y^{\prime}(1)=\beta y^{\prime}(\tau),
\end{array}\right.
$$


respectively with $\alpha=\sum_{i=1}^{m-2} a_{i}, \beta=\sum_{j=1}^{n-2} b_{j}$.

\section{REFERENCES}

[1] Benchohra, M. and Ntouyas, S. K.: A note on a three point boundary value problem for second order differential inclusions, Mathematical Notes, Miskolc, 2(1), (2001), 3947.

[2] Benchohra, M. and Ntouyas, S. K.: On a three and four point boundary value problem for second order differential inclusions, Mathematical Notes, Miskolc, 2(2), (2001), 93-101.

[3] Benchohra, M. and Ntouyas, S. K.: Multi point boundary value problem for second order differential inclusions, Mathematicki Vesnik, 53, (2001), 51-58.

[4] Bitsadze, A. V.: On a class of conditionally solvable nonlocal boundary value problems for harmonic functions, Soviet. Math. Dokl., 31(1), (1985), 91-94.

[5] Bitsadze, A. V. and Samarskit, A. A.: On some simple generalizations of linear elliptic boundary value problem, Soviet. Math. Dokl., 10(2), (1969), 398-400.

[6] Bressan, A. and Colombo, G.: Extensions and selections of maps with decomposable values, Studia Math., 90, (1988), 69-86.

[7] Covitz, H. and NAdleR S. B. JR.: Multivalued contraction mappings in generalized metric spaces, Israel J. Math., 8, (1970), 5-11.

[8] Deimling, K.: Multivalued Differential Equations, Walter de Gruyter, Berlin-New York, 1992.

[9] Frigon, M. and Granas, A.: Théorèmes d'existence pour des inclusions différentielles sans convexité, C. R. Acad. Sci. Paris, Ser. I., 310, (1990), 819-822.

[10] Gorniewicz, L.: Topological Fixed Point Theory of Multivalued Mappings, Mathematics and its Applications, 495, Kluwer Academic Publishers, Dordrecht, 1999.

[11] Gupta, C. P., Ntouyas, S. K. and Tsamatos P. Ch.: On an m-point boundary value problem for second order ordinary differential equations, Nonlinear Anal., 23, (1994), $1427-1436$.

[12] Gupta, C. P., Ntouyas, S. K. and Tsamatos P. Ch.: Existence results for m-point boundary value problems, Differential Equations Dynam. Systems, 2, (1994), 289-298.

[13] Gupta, C. P., Ntouyas, S. K. and Tsamatos P. Сн.: On the solvability of some m-point boundary value problems, Appl. Math., 41, (1996), 1-17.

[14] Gupta, C. P., Ntouyas, S. K. and Tsamatos P. Ch.: Existence results for multi-point boundary value problem for second order ordinary differential equations, Bull. Greek Math. Soc., 43, (2000), 105-123.

[15] Hu, Sh. and Papageorgiou N.: Handbook of Multivalued Analysis, Volume I: Theory, Kluwer Academic Publishers, Dordrecht-Boston-London, 1997.

[16] IL'In, V.A. and Moiseev E.I.: Nonlocal boundary value problem of the first kind for a Sturm-Liouville operator in its differential and finite difference aspects, Differential Equations 23(7), (1987), 803-810.

[17] IL'in, V.A. and Moiseev E.I.: Nonlocal boundary value problem of the second kind for a Sturm-Liouville operator, Differential Equations, 23(8), (1987), 979-987. 
[18] Martelli, M.: A Rothe's type theorem for non-compact acyclic-valued map, Boll. Un. Mat. Ital., 11(3), (1975), 70-76.

[19] Smart D. R.: Fixed Point Theorems, Cambridge Univ. Press, Cambridge, 1974.

[20] Tolstonogov, A. A.: Differential Inclusions in a Banach Space, Kluwer Academic Publishers, Dordrecht, 2000. 